\title{
Application of a Mamdani-Type Fuzzy Rule-Based System to Segment Periventricular Cerebral Veins in Susceptibility-Weighted Images
}

\author{
Francesc Xavier Aymerich ${ }^{1,2}$, Pilar Sobrevilla ${ }^{3}$, Eduard Montseny ${ }^{2}$, Alex Rovira $^{1}$ \\ ${ }^{1}$ MR Unit, Department of Radiology (IDI).Vall d'Hebron University Hospital, Vall d'Hebron \\ Research Institute (VHIR).Autonomous University of Barcelona, 08035 Barcelona, Spain \\ \{xavier.aymerich, alex.rovira\}@idi.gencat.cat
}

${ }^{2}$ ESAII Department, Universitat Politècnica de Catalunya, 08003, Barcelona, Spain

\{xavier.aymerich, eduard.montseny\} @upc.edu

${ }^{3}$ MAII Department, Universitat Politècnica de Catalunya, 08034, Barcelona, Spain

pilar.sobrevilla@upc.edu

\begin{abstract}
This paper presents an algorithm designed to segment veins in the periventricular region of the brain in susceptibility-weighted magnetic resonance images. The proposed algorithm is based on a Mamdani-type fuzzy rule-based system that enables enhancement of veins within periventricular regions of interest as the first step. Segmentation is achieved after determining the cut-off value providing the best trade-off between sensitivity and specificity to establish the suitability of each pixel to belong to a cerebral vein. Performance of the algorithm in susceptibility-weighted images acquired in healthy volunteers showed very good segmentation, with a small number of false positives. The results were not affected by small changes in the size and location of the regions of interest. The algorithm also enabled detection of differences in the visibility of periventricular veins between healthy subjects and multiple sclerosis patients.
\end{abstract}

Keywords. Brain; Fuzzy rule-based systems; Image segmentation; Magnetic resonance imaging

\section{Introduction}

Susceptibility-weighted imaging (SWI) is a noninvasive magnetic resonance imaging (MRI) technique that takes advantage of the magnetic susceptibility effects of paramagnetic deoxygenated hemoglobin [1]. Because of this capability, SWI can be used to visualize venous structures in the brain, providing valuable complementary information for the diagnosis and treatment of patients with neurological disorders such as multiple sclerosis (MS) [2]. A quantitative method to determine the number of veins detected on SWI would be of value for monitoring MS severity, progression, and the response to therapy [3]. 
Segmentation of venous structures over the entire brain with SWI is extremely complex. This is partly because certain regions, including the periventricular white matter, contain numerous small veins, most of them very thin and difficult to differentiate from their surroundings in these sequences. Many of the available methods used for segmenting veins in SW images are adaptations of techniques designed and used for segmenting bright arteries from a dark background [4]. Several methods for cerebrovascular segmentation have been proposed, and a detailed review is provided by Lesage et al. [5]. Focusing only on SWI, two main approaches have been used for segmentation of brain venous structures [6]. The first is based on the use of a statistical local thresholding algorithm [3]. The second approach involves application of scale-space analysis based on vesselness filters, which can be used to directly visualize venous structures [7]. Segmentation is then done using thresholding [8] or an active contour model [9]. Some examples of vesselness filters [10-13] are based on Frangi's [7] and Sato's vesselness filter [8].

Detection of venous blood pixels in SW images addressed to segmenting venous structures in the brain is subject to several factors that imply inherent uncertainty. Most cerebral veins are tiny, thin structures existing in an environment where noise, non-homogeneity, artifacts, and partial volume effects introduce varying degrees of vagueness that affect their detection and the definition of their paths. Fuzzy rule-based systems (FRBSs) are important areas in which fuzzy logic and fuzzy set theory are applied. In contrast to classical rule-based systems, FRBS deal with fuzzy rules instead of classical logical ones, and their success resides in their approximation to human perception and reasoning, and their intuitive handling and simplicity [14].

Some examples of FRBS use in MRI have been reported [15][16], and two studies, conducted by Forkert et al. [17][18], have described FRBS application to solve the problem of segmenting vasculature in MR images. However, Forkert's work focuses on 3D time-of-flight (TOF) magnetic resonance angiography (MRA) rather than magnetic resonance venography with SWI. To our knowledge, the approach presented here is the first application of a FRBS to segment veins in SW images.

\section{$2 \quad$ Materials and methods}

\subsection{Image datasets}

Image datasets for training and test purposes were obtained from 13 healthy individuals (10 women and 3 men), with a mean age of 36.7 years (range, 28-50 years). Images of one of these subjects were used for training and the images of the other 12 were used to test the algorithm. To evaluate the capability of the algorithm to detect differences in vein visibility between patients and healthy subjects, we also studied 13 relapsing-remitting multiple sclerosis (RRMS) patients ( 9 women and 4 men) with a mean age of 37.1 years (range, 28-46 years), mean disease duration of 10.3 years (range, 0.83-22.0 years), and an average Expanded Disability Status Scale (EDSS) score of 3.1. All images were acquired on a Siemens Magnetom Trio 3.0 T scanner (Siemens, Erlangen, Germany) with a 12-channel array head coil using a 3D fast-low angle single shot sequence (repetition time [TR]/echo time [TE], $32 \mathrm{~ms} / 24.6 \mathrm{~ms}$; flip 
angle, $15^{\circ}$; matrix, $320 \times 320$; voxel size, $0.78 \times 0.78 \times 3.0 \mathrm{~mm}^{3}$; iPAT factor, 2). Fiftytwo parallel contiguous axial slices covering the whole brain were acquired using this sequence. The study was approved by the Clinical Research Ethics Committee of Hospital Universitari Vall d'Hebron in Barcelona (Spain).

\subsection{Proposed method}

The proposed algorithm for segmenting veins on SWI has 5 main steps: image selection, preprocessing, definition of regions of interest, enhancement of veins, and segmentation of veins.

The first step is selection of 4 contiguous SW slices from the brain MRI examination of a subject, $I^{n}(1 \leq n \leq 4)$, where the regions of interest can be visualized. Then, structures outside the intracranial region have to be removed in $I^{n}(1 \leq n \leq 4)$ by applying to $I^{n}$ a procedure based on the brain surface extraction (BSE) algorithm [19] included in the Medical Image, Processing, Analysis, and Visualization (MIPAV) software package, version 4.4.1 (Center for Information Technology, NIH, Bethesda, Maryland, USA). Thus, the $I_{B}^{n}(1 \leq n \leq 4)$ images are obtained. These images are then normalized, so that the normalized value for each pixel $p$ located at position $(i, j)$ in $I_{B}^{n}$ is given by:

$$
I_{N}^{n}(i, j)= \begin{cases}\operatorname{int}\left(1000 \frac{I_{B}^{n}(i, j)}{h_{\max }}\right) & \text { if } I_{B}^{n}(i, j) \leq 4 h_{\max } \\ 4000 & \text { otherwise }\end{cases}
$$

where $h_{\max }$ is the gray-level value greater than 150 associated with the maximum frequency in the smoothed histogram $H_{S}\left(I_{B}^{n}\right)$ obtained by averaging the frequency values whose distances in $H\left(I_{B}^{n}\right)$ were less than or equal than 2.

In the next step, pixels belonging to veins are enhanced to best differentiate them from their surroundings within $R O I\left(I_{N}^{n}\right)$ by applying a Mamdani-type FRBS to the images $I_{N}^{n}(1 \leq n \leq 4)$ to obtain the associated Adequacy images, $I_{A}^{n}(1 \leq n \leq 4)$. The inputs of this FRBS are perceptual features of vein pixels within ROIs evaluated with low-level operators, and the output is the adequacy of these pixels to belong to veins.

Finally, a cut-off value is applied to the Adequacy images to obtain the segmented images, $I_{S V}^{n}(1 \leq n \leq 4)$, whose value for a pixel $p$ located at position $(i, j)$ equals one if it belongs to $\operatorname{ROI}\left(I_{N}^{n}\right)$ and $I_{A}^{n}(i, j)>c$, and is zero otherwise. ROC curve analysis was used to select the best cut-off value.

\subsection{Implementation of the method}

This section describes how the algorithm steps were implemented. 


\section{Selection of images, preprocessing, and definition of ROIs}

From the 52 axial slices acquired in the examination of one healthy volunteer, we selected 4 contiguous axial slices, $I_{t}^{n}(1 \leq n \leq 4)$, where the ROIs were visualized. The training images $I_{t}^{n}$ were then normalized following the preprocessing procedure previously described, obtaining $I_{N t}^{n}$. In each hemisphere of $I_{N t}^{n}$ we then manually defined a $9.38 \mathrm{~mm}$ by $42.97 \mathrm{~mm}$ rectangular ROI in the periventricular region mainly occupied by white matter from the corona radiata, $\operatorname{ROI}\left(I_{N_{t}}^{n}\right)$. The pixels within the 8 rectangular ROIs obtained are the only ones included in the next steps.

\section{Enhancement of veins within the ROIs}

\section{Selection of features}

A look inside the white rectangles in Fig. 1(a) shows that veins in SW images are visualized as mainly linear structures showing a darker gray level than their immediate surroundings. Comparison of the linear venous structures in the ROIs and the dark structures within the ellipses of the magnified image in Fig. 1(b) shows that the veins are lighter than the wider vessels in other locations, and some of them show short discontinuities. In addition, certain thin, dark structures are seen outside the ROIs, such as areas of cortex with a high iron content (Fig. 1b, white arrows), whose characteristics may cause them to be mistaken for veins.

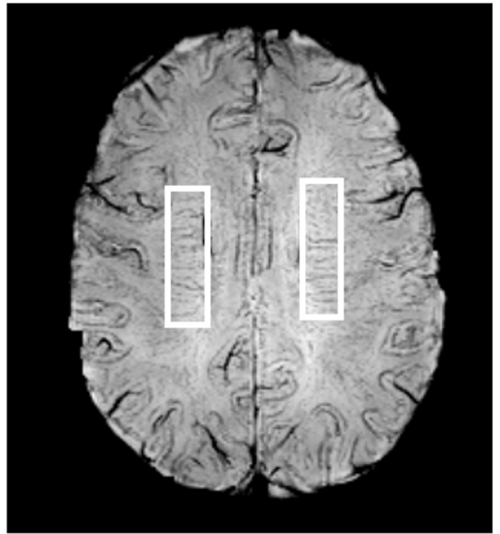

(a)

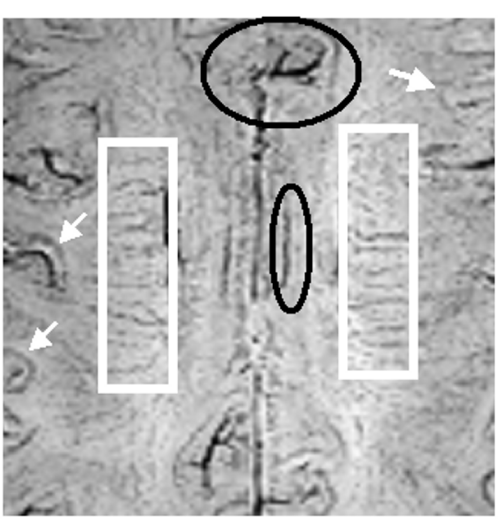

(b)

Fig. 1. Example of SW image after application of the BSE algorithm and normalization process. (a) Location of ROIs (white rectangles). (b) Magnification of the area containing ROIs in which black ellipses enclose blood vessels showing better differentiation than most of those within the ROIs, and white arrows point to some cortex locations with high iron content, which, because of their perceptual features, could be mistaken for vein vessels. 
Taking into consideration these factors, 3 features are essential to detect vein pixels within periventricular ROIs: Gray-level, Thinness, and Linearity.

Selection of operators to evaluate the features

Several low-level operators were analyzed, seeking those that best characterized Gray-level, Thinness, and Linearity. Selection of the most appropriate operator to evaluate Gray-level involved analysis of 9 operators: gray-level of the central pixel, and maximum, minimum, mean and median gray-level values of the pixels covered by $3 \times 3$ and $5 \times 5$ raster windows. The standard deviations of the gray-level values within these windows and the gray-level differences between the central pixel and its neighbors were the 26 operators analyzed for Thinness. Lastly, to select the operator to evaluate Linearity, we used kernels that enabled detection of horizontal, vertical, or oblique ( +45 and -45 degrees) single-pixel-wide lines. Application of these kernels required inverting the gray scale of the images in order to detect dark linear structures.

To obtain the operators, pixels corresponding to veins located within the ROIs in the training set images $I_{N t}^{n}(1 \leq n \leq 4), R O I\left(I_{N t}^{n}\right)$, were manually labeled using MRIcro software [20]. These were the reference images $I_{R E F V t}^{n}(1 \leq n \leq 4)$, in which the value assigned to a pixel in the $(i, j)$ position was 1 if it belonged to a vein and 0 otherwise. Moreover, since our analysis was focused on finding the best operators to evaluate the features of vein pixels, pixels within $I_{R E F V}^{n}$ were divided into 3 reference sets: $S_{l}$, comprising pixels labeled as veins and belonging to very thin veins; $S_{2}$, including pixels labeled as veins without thinness restrictions; and $S_{3}$, comprising pixels that were not labeled.

The operator selected to evaluate each feature had to maximize the separability, defined by the ratio between the absolute value of the mean value difference and the maximum of standard deviation values obtained for the reference sets $S_{r}$ and $S_{s}(r, s \in$ $\{1,2,3\}, r \neq s)$. Then, the operators showing the best performance for evaluating the features of each pixel $p$ located at $(i, j)$ were as follows: gray-level value, $g l(i, j)$, for the Gray-level feature; the third highest difference between the central and surrounding pixels within a $5 \times 5$ window centered on the pixel, $\operatorname{dif}_{5 x 5}^{3}(i, j)$, for the Thinness feature; and the maximum of 4 directional $3 \times 3$ kernels centered on $(i, j), \max K 3 \times 3(i, j)$, for the Linearity feature.

\section{Proposed Mamdani-type FRBS}

The principal elements of the FRBS were defined and designed based on a priori knowledge, in this case, expert judgment and experience. The underlying knowledge of the system was then explicitly translated into a set of easy to interpret linguistic labels using fuzzy rules.

Knowledge Base: The data base (DB) of the proposed system is comprised of sets of linguistic terms and the membership function partitions associated with the three input variables introduced in previous section (Gray-level, Thinness, and Linearity), and the output variable (Adequacy of a pixel to belong to a venous blood vessel). The sets of linguistic terms considered for the input and output variables are given as follows: 


$$
\begin{aligned}
& L_{\text {Gray-level }}=\{\text { Dark, Medium-Dark, Light }\}=\{D, M D, L G\} \\
& L_{\text {Thinness }}=\{\text { Low, Medium, High }\}=\left\{L_{\text {thin }}, M_{\text {thin }}, H_{\text {thin }}\right\} \\
& L_{\text {Linearity }}=\{\text { Low, Medium, High }\}=\left\{L_{\text {lin }}, M_{\text {lin }}, H_{\text {lin }}\right\} \\
& L_{\text {Adequacy }}=\{\text { Very Poor, Poor, Fair, Good, Excellent }\}=\{V P, P, F, G, E\}
\end{aligned}
$$

To define the semantics of these linguistic labels, we adopted trapezoidal-shaped membership functions (MFs). The MFs were defined by the quadruple $(s l, c l, c u, s u)$, where $s l$ and $s u$, and $c l$ and $c u$ are the lower and upper bounds of the support and the core, respectively. The shapes of the MFs can be seen in Figs. 2, which show the fuzzy partitions defined on the domains of each variable.

MFs associated with the input variables were obtained by analyzing the values of the features within and around $\operatorname{ROI}\left(I_{N t}^{n}\right)$ defined in $I_{N t}^{n}(1 \leq n \leq 4)$, whereas the uniform partition corresponding to the output variable involved 5 levels of adequacy.
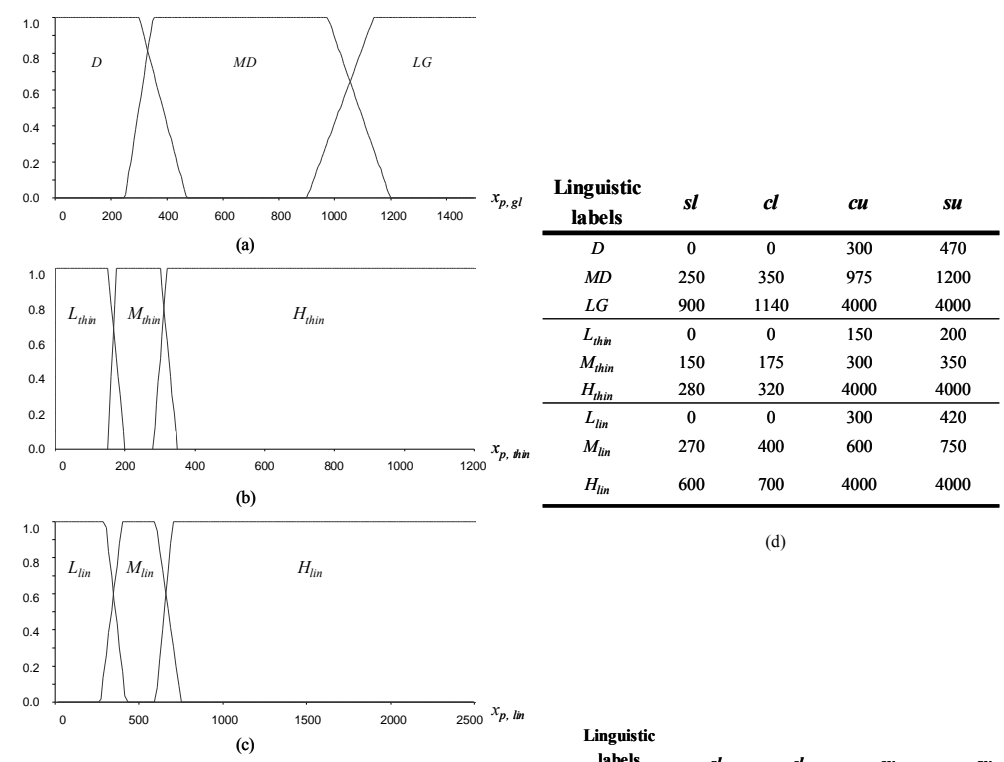

(d)

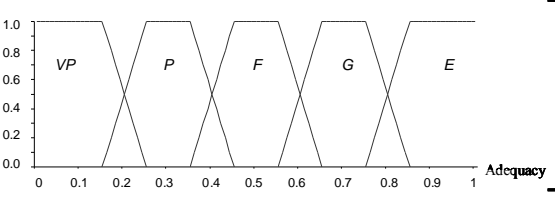

\begin{tabular}{ccccc}
$\begin{array}{c}\text { Linguistic } \\
\text { labels }\end{array}$ & $s l$ & $c l$ & $c u$ & $s u$ \\
\hline$V P$ & 0.0 & 0.0 & 0.15 & 0.25 \\
$P$ & 0.15 & 0.25 & 0.35 & 0.45 \\
$F$ & 0.35 & 0.45 & 0.55 & 0.65 \\
$G$ & 0.55 & 0.65 & 0.75 & 0.85 \\
$E$ & 0.75 & 0.85 & 1.0 & 1.0 \\
\hline
\end{tabular}

(f)

Fig. 2. Trapezoidal-shaped membership functions defining the semantics of the linguistic labels. Input variables associated with (a) Gray-level, (b) Thinness, and (c) Linearity features. (d) Output variable associated with Adequacy. Values defining the shape of each membership function are given in (d) for input variables, and in (f) for the output variable. 
The rule base $(\mathrm{RB})$ is represented in compact format by the decision table shown in Table 1. To achieve good performance, the 27 rules conforming the RB were obtained taking into account expert human knowledge and attending to a trade-off between the number of pixels associated with true and false positives. The pixel values for the features fire each rule for the eight ROIs defined in the images of $\operatorname{ROI}\left(I_{N t}^{n}\right)$.

\begin{tabular}{ccccc}
\hline \multirow{4}{*}{ Gray-Level } & Thinness & \multicolumn{3}{c}{ Linearity } \\
\cline { 3 - 5 }$D$ & $L_{\text {lin }}$ & $M_{\text {lin }}$ & $H_{\text {lin }}$ \\
\hline \multirow{4}{*}{$D$} & $L_{\text {thin }}$ & $V P$ & $V P$ & $V P$ \\
& $M_{\text {thin }}$ & $V P$ & $V P$ & $P$ \\
& $H_{\text {thin }}$ & $V P$ & $P$ & $F$ \\
\hline \multirow{4}{*}{$M D$} & $L_{\text {thin }}$ & $F$ & $G$ & $F$ \\
& $M_{\text {thin }}$ & $F$ & $E$ & $E$ \\
& $H_{\text {thin }}$ & $P$ & $F$ & $G$ \\
\hline \multirow{4}{*}{$L G$} & $L_{\text {thin }}$ & $P$ & $F$ & $P$ \\
& $M_{\text {thin }}$ & $P$ & $F$ & $F$ \\
& $H_{\text {thin }}$ & $V P$ & $P$ & $P$ \\
\hline
\end{tabular}

Table 1. Decision table describing the rule base of the proposed FRBS

Inference Engine: The fuzzification interface establishes a mapping from crisp input values to fuzzy sets defined in the universe of discourse of this input, $U=U_{g l} \times U_{\text {thin }}$ $\mathrm{x} U_{l i n}$. To do so, for each pixel $p$ belonging to an ROI we obtained the corresponding input vector: $x_{p}=\left(x_{g l}, x_{t h i n}, x_{l i n}\right)=\left(g l(i, j), \operatorname{dif}_{5 x 5}^{3}(i, j), \operatorname{maxK} 3 x 3(i, j)\right)$. Then, we sought the degree to which it belonged to each of the fuzzy sets defined in $U$; that is, $\mu_{D}\left(x_{g l}\right)$, $\mu_{M D}\left(x_{g l}\right), \mu_{L G}\left(x_{g l}\right), \mu_{L, \text { thin }}\left(x_{\text {thin }}\right), \mu_{M, \text { thin }}\left(x_{\text {thin }}\right), \mu_{H, \text { thin }}\left(x_{\text {thin }}\right), \mu_{L, \text { lin }}\left(x_{\text {lin }}\right), \mu_{M, l i n}\left(x_{\text {lin }}\right), \mu_{H, \text { lin }}\left(x_{\text {lin }}\right)$, which were obtained via the corresponding trapezoidal membership functions.

The inference system and defuzzification interface selected to derive the fuzzy and crisp outputs were the classical ones used by Mamdani [21]. These involve the minimum $t$-norm for both the conjunctive operator, $T$, which derives the rules firing according to the decision table, and the implication operator, $I$, which determines the output of the compositional rule of inference. Mode A-FATI is the defuzzification interface, where the aggregation operator, $G$, is modeled by the maximum $t$-conorm, while the defuzzification method, $D$, is the center of gravity (COG), such that:

$$
\begin{gathered}
\mu_{A_{k}}\left(x_{p}\right)=\operatorname{Min}\left(\mu_{A_{k, g l}}\left(x_{g l}\right), \mu_{A_{k, \text { thin }}}\left(x_{\text {thin }}\right), \mu_{A_{k, \text { lin }}}\left(x_{\text {lin }}\right)\right), k=1, \ldots, 27 \\
\mu_{B_{k}^{\prime}}(y)=\operatorname{Min}\left(\mu_{A_{k}}\left(x_{p}\right), \mu_{B_{k}}(y)\right), k=1, \ldots, 27 \\
\mu_{B^{\prime}}(y)=\operatorname{Max}_{1 \leq k \leq 27}\left(\mu_{B_{k}^{\prime}}(y)\right) \\
y_{p}=\operatorname{COG}\left(\mu_{B^{\prime}}(y)\right)=\frac{\int_{Y} y \mu_{B^{\prime}}(y) d y}{\int_{Y} \mu_{B^{\prime}}(y) d y} \approx \frac{\sum_{i=1}^{N} y_{i} \mu_{B^{\prime}}\left(y_{i}\right)}{\sum_{i=1}^{N} \mu_{B^{\prime}}\left(y_{i}\right)}
\end{gathered}
$$


where $y_{i}$ was the i-value in the uniform partition of the adequacy interval $[0,1]$ in $\mathrm{N}$ values, where the value of $\mathrm{N}$ was 100 . The values $y_{p}$ so obtained provided the adequacy of each pixel $p$ in $R O I\left(I_{N t}^{n}\right)$ to be a vein pixel.

\section{Segmentation of veins}

After applying the FRBS to the training images, $I_{N t}^{n}(1 \leq n \leq 4)$, we obtained the associated Adequacy images, $I_{A t}^{n}(1 \leq n \leq 4)$ : For each pixel $p$, the value of $I_{A t}^{n}(p)$ was the value obtained if the pixel belonged to the ROI. If not, the value was set at zero.

We then sought the cut-off value, " $c$ ", providing the best segmentation results. We considered 71 cut-off values, $c_{i}(1 \leq i \leq 71)$, in the interval [0.2, 0.9] and applied them to the Adequacy images, $I_{A t}^{n}(1 \leq n \leq 4)$. For each $c_{i}$, four segmented images were obtained, $I_{S V t}^{n}(1 \leq n \leq 4)$, whose value for a pixel $p$ located at position $(i, j)$ equaled one if it belonged to $\operatorname{ROI}\left(I_{N t}^{n}\right)$ and $I_{A t}^{n}(i, j)>c_{i}$, and was zero otherwise. To know the number of true positives (TP), false positives (FP), true negatives (TN), and false negatives (FN), a pixel-by-pixel comparison was performed between $I_{S V t}^{n}$ and the reference image, $I_{R E F V t}^{n}$, manually labeled by an expert to determine the correctness of the classification within $R O I\left(I_{N t}^{n}\right)$.

To select the best cut-off value, we used an ROC plot, representing the trade-off between sensitivity (S), that is the true positive rate (TPR), and the false positive rate (FPR), for each $c_{i}$ value. The true positive and false positive rates are given by $\mathrm{S}=\mathrm{TPR}=\mathrm{TP} /(\mathrm{TP}+\mathrm{FN})$, and $\mathrm{FPR}=\mathrm{FP} /(\mathrm{TN}+\mathrm{FP})$. Applying this process, we obtained 71 points (FPRci, TPRci), depicted in the ROC space. The best trade-off value is defined by the closest point to the upper left corner, located at $(0.0316,0.9394)$, which in our case was $\mathrm{c}=0.58$.

\section{$3 \quad$ Results}

To evaluate the performance of the algorithm, we selected quality indices that enabled pixel-by-pixel comparison within the ROIs of the reference and segmented images. The indices were also defined based on the number of TP, FP, TN, and FN. Then, in addition to the true and false positive rates we also considered specificity (SPC), accuracy (ACC), and the Dice coefficient (DC). These quality indices are defined by $\mathrm{SPC}=\mathrm{TN} /(\mathrm{TN}+\mathrm{FP}), \mathrm{ACC}=(\mathrm{TP}+\mathrm{TN}) /(\mathrm{TP}+\mathrm{FP}+\mathrm{TN}+\mathrm{FN})$, and $\mathrm{DC}=2 \mathrm{TP} /(2 \mathrm{TP}+\mathrm{FP}+\mathrm{FN})$.

\subsection{Evaluation of the algorithm on training and test images}

\section{Training images}

The results for the training images are presented in the third row of Table 2, which shows the mean $(\mathrm{m})$ and standard deviation $(\sigma)$ values for the indices S, SPC, ACC, 
and DC in the comparison between the segmented and reference images. As was expected, and as can be inferred from the values greater than 0.9 obtained for sensitivity and Dice coefficient (third and sixth columns in Table 2), the vast majority of pixels labeled as veins within the reference images were detected by the algorithm. Moreover, the specificity and accuracy values (fourth and fifth columns of Table 2) point to a low number of false positives. Finally, the low standard deviation values with regard to the mean values obtained for all the indices studied indicate that there were no large differences in terms of detection quality between the regions analyzed.

\begin{tabular}{|c|c|c|c|c|c|}
\hline Algorithm & Group & $\begin{array}{c}\mathrm{S} \\
m(\sigma)\end{array}$ & $\begin{array}{c}\mathrm{SPC} \\
m(\sigma)\end{array}$ & $\begin{array}{l}\mathrm{ACC} \\
m(\sigma)\end{array}$ & $\begin{array}{c}\mathrm{DC} \\
m(\sigma)\end{array}$ \\
\hline Proposed & Training & $0.938(0.017)$ & $0.968(0.001)$ & $0.962(0.005)$ & $0.916(0.022)$ \\
\hline Frangi's & $-\underbrace{\text { Test }}_{\text {Test }}$ & $\frac{0.914}{0.776}(0.044)$ & $-\frac{0.969}{(0.023)}$ & $\frac{0.957}{0.847} \frac{(0.017)}{(0.028)}$ & $\frac{0.910(0.031)}{0.707(0.031)}$ \\
\hline Sato's & Test & $0.796(0.054)$ & $0.866(0.045)$ & $0.851(0.029)$ & $0.720(0.029)$ \\
\hline
\end{tabular}

Table 2. Quality index values obtained with the proposed algorithm and other approaches

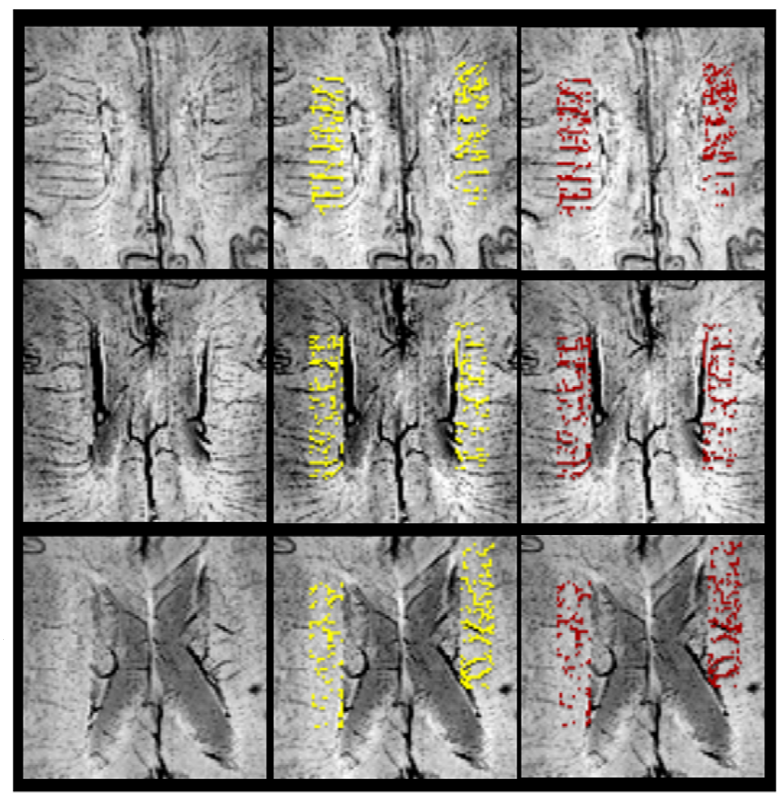

(a)

(b)

(c)

Fig. 3. Examples of the results obtained by processing three test slices corresponding to different anatomical locations. (a) Magnification of the original image area including ROIs. Columns (b) and (c) show overlays of the reference delineated and the segmentation results, respectively 


\section{Test images}

To help in the interpretation of the results for the 96 regions of interest, $\operatorname{ROI}\left(I_{N T}^{n}\right)$, selected in the test images, the fourth row of Table 2 shows the mean $(m)$ and standard deviation $(\sigma)$ for S, SPC, ACC, and DC. As can be seen, the mean values for the test and training images are similar for all the indices except sensitivity, which has a slightly lower mean value for the test images. Furthermore, the $\sigma$ values for the test images are somewhat higher than those for the training images, but this is because of a greater data heterogeneity from the test set images. Therefore, despite the higher $\sigma$ values, the results indicate a similar performance for the test and training images.

Figure 3 illustrates the performance of the algorithm. Column (a) is a magnified depiction of $I_{N T}^{n}$ around $R O I\left(I_{N T}^{n}\right)$, the overlays in column (b) correspond to the manual marking of vein pixels by an expert, $I_{R E F V T}^{n}$, and the overlays in column (c) are the results of vein segmentation, $I_{S V T}^{n}$. The vast majority of pixels labeled as veins in the reference images were included in the segmentation. Only a very small number of false positives and false negatives occurred (see quality indices in Table 2). The false positives were mainly due to factors such as movement during image acquisition, small differences in the width of some vessels between the reference and segmented images, and visualization of other thin structures rich in iron. The false negatives were mainly due to partial volumes in some locations within the ROIs.

\subsection{Dependence on the window properties}

Location and size of the window selected for ROI delineation must be taken into account to avoid false positives. Structures surrounding periventricular white matter may have characteristics that make them difficult to differentiate from veins when they are partially included in ROIs and an automatic segmentation is applied.

\begin{tabular}{ccccc}
\hline & S & SPC & ACC & DC \\
Window & $m(\sigma)$ & $m(\sigma)$ & $m(\sigma)$ & $m(\sigma)$ \\
\hline$W_{l}$ & $0.914(0.044)$ & $0.961(0.023)$ & $0.957(0.017)$ & $0.910(0.030)$ \\
$W_{2}$ & $0.916(0.044)$ & $0.970(0.023)$ & $0.958(0.018)$ & $0.913(0.031)$ \\
$p$-value & 0.827 & 0.839 & 0.822 & 0.643 \\
\hline
\end{tabular}

Table 3. Quality index values obtained for test images using windows: $W_{l}$ and $W_{2}$.

The previous analysis was done using ROIs defined by $9.38 \mathrm{~mm}$ x $42.97 \mathrm{~mm}$ rectangular windows $\left(W_{l}\right)$. As an initial approach to evaluate the influence of the size and position of the window, we tested a smaller $(8.59 \mathrm{~mm} \times 39.06 \mathrm{~mm})$ window $\left(W_{2}\right)$. Although the size of $W_{2}$ was not greatly different from $W_{1}$, the use of a smaller window helped to select positions with a lower possibility of presenting FP.

To evaluate the effect of using one or another window in the test images, we compared the quality indices obtained when ROIs were derived using $W_{1}$ or $W_{2}$ (Table 3). Means and standard deviations for S, SPC, ACC, and DC were very similar using the two windows, and p-values for the differences (bottom row) were not significant. 


\subsection{Comparison with other methods}

To assess the gain of the proposed approach, we compared it with two publicly available methods developed for problems similar to the one proposed here. These two approaches were based on implementation of vesselness enhancement filters (VEF), developed by Frangi [7] and Sato [8], and included in the VMTK module [22] of the 3Dslicer software [23]. First, the optimum values for the parameters of the approaches based on these filters were selected by evaluation of their segmentation performance on $R O I\left(I_{N t}^{n}\right)$. We then used these approaches to analyze $R O I\left(I_{N T}^{n}\right)$.

The results obtained using the filters on $\operatorname{ROI}\left(I_{N T}^{n}\right)$ are shown in Table 2. Although the approaches based on the VEF yielded high values for the four quality indices, the proposed approach showed better performance: a significant improvement $(p<0.001$, ANOVA test) of around $10 \%$ to $15 \%$ in S, SPC, and ACC, and around $26 \%$ in DC.

\subsection{Evaluation of the ability to detect changes in the visibility of veins}

To evaluate the applicability of the proposed Mamdani-type FRBS algorithm for clinical purposes, we considered an approach similar to that reported by Ge et al. [3]. The authors demonstrated a significant reduction in the visibility of veins in the periventricular white matter in RRMS patients compared to controls.

In this evaluation, we included images from 13 RRMS patients and 13 healthy controls. The proposed FRBS was applied to the ROIs defined in these images, and the segmented images were obtained. Following segmentation, the mean number of venous blood voxels within the ROIs was $108.60(\sigma=14.70)$ in RRMS patients and $126.94(\sigma=18.70)$ in healthy controls, and there exists a significant difference ( $\mathrm{p}=0.01$, Student's $t$-test) between these two groups, as was reported by Ge et al. [3].

\section{Conclusion}

The algorithm proposed here is based on a fuzzy rule-based system that allows segmenting periventricular venous vasculature in SW MR images. The algorithm entails initial enhancement of cerebral veins in defined ROIs using an FRBS to determine the adequacy of each pixel to belong to a vein, and applies a cut-off value for final segmentation of these structures. The results obtained from healthy volunteers showed very good segmentation with a very small number of false positives. The method also proved to be robust and applicable to the study of periventricular veins in MS.

\section{References}

1. Haacke EM, Xu YB, Cheng YCN, Reichenbach JR. Susceptibility weighted imaging (SWI). MRM 52 (2004) 1-40.

2. Mittal S, Wu Z, Neelavalli J, Haacke EM. Susceptibility-weighted imaging: Technical aspects and clinical applications, Part 2, Am. J. Neuroradiol. 30 (2009) 232-252. 
3. Ge Y, Zohrabian VM, Osa E, et al. Diminished visibility of cerebral venous vasculature in MS by susceptibility-weighted imaging at 3.0 Tesla. JMRI 29 (2009) 1190-1194.

4. Suri JS, Liu KC, Reden L et al.. A review on MR vascular image processing: skeleton versus nonskeleton approaches: Part II, IEEE Trans. on IT in Biomed 6 (2002) 338-350.

5. Lesage D, Angelini ED, Bloch I, Funka-Lea G. A review of 3D vessel lumen segmentation techniques: Models, features and extraction schemes. MIA 13 (2009) 819-845.

6. Haacke EM, Reichenbach JR. Susceptibility weighted imaging in MRI: basic concepts and clinical applications. Willey-Blackwell; 2011.

7. Frangi AF, Niessen WJ, Hoogeveen RM et al. Model-based quantitation of 3-D magnetic resonance angiographic images. IEEE Trans. on Med Imaging 18 (1999) 946-956.

8. Sato Y, Nakajima S, Shiraga N, et al. 3D multi-scale line filter for segmentation and visualization of curvilinear structures in medical images. MIA 2 (1998) 143-168.

9. Lorenz C, Carlsen IC, Buzug TM et al. A multi-scale line filter with automatic scale selection based on the hessian matrix for medical image segmentation, In: Scale-Space. Theory in Computer Vision, ter Haar Romeny B, Florack L, Koenderink J et al, Ed. 1997 152-163.

10. Grabner G, Dal-Bianco A, Hametner S et al. Group specific vein-atlasing: an application for analyzing the venous system under normal and MS conditions. JMRI 40 (2014) 655-61

11. Manniesing R, Viergever MA, Niessen WJ. Vessel enhancing diffusion - A scale space representation of vessel structures. MIA 10 (2006) 815-825.

12. Koopmans PJ, Manniesing R, Niessen WJ et al. MR venography of the human brain using susceptibility weighted imaging at very high field strength. MAGMA 21 (2008) 149-158.

13. Zivadinov R, Poloni GU, Marr K, et al. Decreased brain venous vasculature visibility on susceptibility-weighted imaging venography in patients with multiple sclerosis is related to chronic cerebrospinal venous insufficiency. BMC Neurology 11(2011) 128. Available: http://www.biomedcentral.com/1471-2377/11/128.

14. Nauck D, Kruse R. Obtaining interpretable fuzzy classification rules from medical data. AIM 16 (1999) 149-169.

15. Mehta SB, Chaudhury S, Bhattacharyya A, Jena A. Soft-computing based diagnostic tool for analyzing demyelination in magnetic resonance images. ASOC 10 (2010) 529-538.

16. Zarandi MHF, Zarinbal M, Izadi M. Systematic image processing for diagnosing brain tumors: A type-II fuzzy expert system approach. ASOC 11 (2011) 285-294.

17. Forkert ND, Schmidt-Richberg A, Fiehler J, et al. Fuzzy-based vascular structure enhancement in Time-of-Flight MRA images for improved segmentation. Methods of Information in Medicine 50 (2011) 74-83.

18. Forkert ND, Schmidt-Richberg A, Fiehler J et al. 3D cerebrovascular segmentation combining fuzzy vessel enhancement and level-sets with anisotropic energy weights. MRI 31 (2013) 262-271.

19. Shattuck DW, Sandor-Leahy SR, Schaper KA et al. Magnetic resonance image tissue classification using a partial volume model. Neuroimage 13 (2001) 856-876.

20. Rorden C, Brett M. Stereotaxic display of brain lesions. Behav Neurol 12 (2000) 191-200.

21. Mamdani EH. Application of fuzzy algorithms for control of simple dynamic plant. Proceedings of the Institution of Electrical Engineers-London 121 (1974) 1585-1588.

22. Haehn, D. The Vascular Modeling Toolkit in 3D Slicer (Modules:VMTKVessel Enhancement) (2010). http://www.slicer.org/slicerWiki/index.php/Modules: VMTKVesse1Enhancement (accessed March 27, 2015).

23. Pieper S, Lorensen B, Schroeder W, Kikinis R. The NA-MIC kit: ITK, VTK, pipelines, grids and $3 \mathrm{D}$ slicer as an open platform for the medical image computing community. In: 3rd IEEE Int. Symp.on Biomedical Imaging: Macro to Nano 1-3 (2006) 698-701. 